\title{
Statistical Performance Analyzer for Compressive Sensing Gradient Algorithm
}

\author{
Stefan Vujović, Irena Orović, Miloš Daković, Ljubiša Stanković \\ Faculty of Electrical Engineering, University of Montenegro \\ Podgorica, Montenegro \\ stefanv@ac.me
}

\begin{abstract}
The paper presents a statistical performance analyzer for compressive sensing gradient algorithm. It is composed of two main parts. The first part is designed as a tool for signal reconstruction based on the gradient algorithm. The possibility to generate various signals is incorporated through the special panel which is used to set the length, sparsity, percent of available samples and the ranges of amplitude. Also, a noise with specified variance can be added through this panel as well. The second part of presented instrument is dedicated to the statistical analysis. Different analysis can be performed through this part such as the calculation of MSE as a function of noise variance or signal sparsity, as well as the analysis of MSE and computational time in terms of sparsity and the amount of missing samples. This instrument can be useful for both the research and educational purpose for the area of compressive sensing and signal reconstruction.
\end{abstract}

Keywords-Statistical Analyzer; Gradient Algorithm; Compressive Sensing; Sparse signal processing

\section{INTRODUCTION}

Signals that can be represented by a small number of nonzero coefficients compared to the signal length are called sparse in the observed representation basis. Signal having sparse presentation in one domain is usually dense in some other domain. For example, each pixel of image usually has non-zero value. However, the Discrete cosine transform (DCT) for most of the pictures has small number of non-zero coefficients while the rest of them can be neglected without loosing the image quality. Thus, we can say that DCT is sparse domain for most of the images. The sparsity domain can be also any other transform domain such as Discrete Fourier transform (DFT), Discrete wavelet domain (DWT), etc. Sparse signals are of interest in many practical applications like radars, sonars, biomedicine, etc. The area dealing with sparse signals and its reconstruction from an incomplete set of samples is called Compressive sensing (CS) and it has been of great interest in the last decade [1], [2].

The idea behind the CS concept is to reduce sampling rate compared to the conventional one defined by Nyquist theorem. It was shown that the signals having sparse presentation in certain domain can be reconstructed from reduced set of linear measurements. The number of measurements that is sufficient to reconstruct the whole signal is related to the sparsity of considered signal. For lower level of sparsity, a smaller number of measurements is required. Theory of CS is studied in [3-5], and there are two main research directions there. The first one is about sensing matrix which makes relation between $\mathrm{N}$ samples of signal in dense domain and its $M(M \ll N)$ linear measurements. The second one is about reconstruction of the original signal of length $N$ from its $M$ linear measurements. There are many algorithms dealing with this kind of problem, and among them we may distinguish two major classes: convex relaxations algorithms and greedy algorithms (based on matching pursuit, thresholding, etc) [6], [7].

This paper brings the realization of the virtual instrument and analyzer for recently proposed gradient algorithm [8] belonging to convex class of algorithms. The instrument is designed to facilitate the use and analysis of the mentioned algorithm in many different situation. It allows to design different signals by specifying the level of sparsity, the number of measurements, the components amplitudes, etc. Also, input noise can be added in order to test and analyze algorithm since most of the algorithms do not perform very well in this case. The second part of instrument is statistical performance analyzer. Here, we can perform different statistical analysis and obtained results are averaged over given number of iterations and shown graphically. Parameter of interest here is MSE and it is presented as a functions of sparsity or input noise variance. Also, MSE and computational time are presented as a functions of sparsity and number of missing samples.

The paper is organized as follows. After introduction, the theoretical background about CS is provided in Section II. Section III is related to the gradient algorithm. The proposed virtual instrument is described in Section IV. The conclusion is given in Section V.

\section{THEORETICAL BACKGROUND}

Consider discrete-time signal $x(n)$, where $n=0,1, \ldots, N-1$. This signal might have sparse presentation in various domains. Here, we will consider the DFT as a sparse domain of signal. A vector of time domain signal samples will be denoted by $\mathbf{x}$. The FT transformation of considered signal will be denoted by $X(k)$, where $k=0,1, \ldots, N-1$, and vector presentation of this signal will be $\mathbf{X}$. The relations between signal $\mathbf{x}$ and its transform $\mathrm{X}$ can be expressed by: 


$$
\begin{aligned}
\mathbf{X} & =\mathbf{W} \mathbf{x} \\
\mathbf{x} & =\mathbf{W}^{-1} \mathbf{X}
\end{aligned}
$$

where $\mathbf{W}$ is $N \times N$ DFT matrix with elements $W(n, k)=$ $\exp (-2 j \pi n k / N)$.

Let suppose that signal $\mathrm{x}$ have only $M(M<<N)$ available samples, while the other $N-M$ samples are missing or are unavailable. Vector of available samples $y(i)=x\left(n_{i}\right)$, where $i=1, \ldots, M$ will be denoted as $\mathbf{y}$. These available samples are actually linear measurements of signal $\mathbf{X}$ and matrix $\mathbf{A}$ obtained from matrix $\mathbf{W}^{-1}$ by eliminating rows corresponding to missing samples. This can also be expressed in matrix notation as $\mathbf{y}=\mathbf{A X}$. As we can see, vector $\mathbf{y}$ is obtained as a linear combination of elements from the vector $\mathbf{X}$

Under assumption that signal $\mathbf{X}$ is sparse, the CS theory states that whole signal $\mathbf{X}$ can be reconstructed from $M$ samples of $\mathbf{y}$. By reconstructing $\mathbf{X}$ we can uniquely determine $\mathbf{x}$, since they present the same signal in two transformation domains. Thus, the reconstruction task can be defined as:

$$
\tilde{\mathbf{X}}=\arg \min \|\mathbf{X}\|_{0} \text { subject to } \mathbf{y}=\mathbf{A X} \text {. }
$$

Here, the optimal solution would be the sparsest vector satisfying the linear system of equations. However, solving the problem using the $l_{0}$-norm minimization is NP hard combinatorial approach. Moreover, in practical signal processing applications, $l_{0}$-norm is sensitive to very small values even of computer precision order [9]. It is the reason why $l_{1}$-norm minimization is commonly used. The reconstruction task can be formulated as:

$$
\tilde{\mathbf{X}}=\arg \min \|\mathbf{X}\|_{1} \text { subject to } \mathbf{y}=\mathbf{A X} \text {. }
$$

It is very important to note that solutions of (3) and (4) are the same if matrix A satisfy reconstructed isometry properties (RIP) [10].

After sparse signal $\mathbf{X}$ is approximated by (4), a reconstructed signal is obtained as $\mathbf{x}_{\mathbf{r}}=\mathbf{W}^{-1} \mathbf{X}$.

\section{GRAdiEnt Algorithm}

Recently proposed gradient algorithm [8] for sparse signal reconstruction belongs to class of convex optimization algorithms. The idea behind this algorithm is to reconstruct time domain samples that are missing by minimizing concentration in sparse domain. Concentration in sparse domain can be measured in various ways, but $l_{1}$-norm is commonly used. Algorithm will be explained in sequel. The sparse domain will be DFT as it was mentioned before. We will review algorithm from [8] next.

Consider a discrete-time signal $x(n)$ of length $N$ with some samples that are missing or are not available. Missing samples positions are denoted by $n_{i}$, where $i=1,2, \ldots, M$, and $M$ is total number of missing samples. Assume that signal is sparse in a DFT domain.
Step 0: Form the initial signal $y^{(0)}(n)$, where (0) means that it is initial state (0 iteration) of algorithm, as:

$$
y^{(0)}(n)= \begin{cases}x(n) & \text { for available samples } \\ 0 & \text { for missing samples }\end{cases}
$$

Step 1: For each missing sample at $n_{i}$ we form two signals $y_{1}(n)$ and $y_{2}(n)$ in each next iteration as

$$
\begin{aligned}
& y_{1}^{(k)}(n)= \begin{cases}y^{(k)}(n)+\Delta & \text { for } n=n_{i} \\
y^{(k)}(n) & \text { for } n \neq n_{i}\end{cases} \\
& y_{2}^{(k)}(n)= \begin{cases}y^{(k)}(n)-\Delta & \text { for } n=n_{i} \\
y^{(k)}(n) & \text { for } n \neq n_{i}\end{cases}
\end{aligned}
$$

where $k$ is the iteration number. Constant $\Delta$ is used to determine whether the considered signal sample should be decreased or increased.

Step 2: Estimate the differential of the signal transform measure as

$$
g\left(n_{i}\right)=\frac{\sum\left|\operatorname{DFT}\left[y_{1}^{(k)}(n)\right]\right|-\sum\left|\operatorname{DFT}\left[y_{2}^{(k)}(n)\right]\right|}{N},
$$

The differential of measure is proportional to the error $\left(y^{(k)}(n)-x(n)\right)$.

Step 3: Form a gradient vector $\mathbf{G}$ with the same length as the signal $x(n)$. At positions of available samples, this vector has value $G(n)=0$. At the positions of missing samples it has values $g\left(n_{i}\right)$ calculated by (8).

Step 4: Update the values of signal $y(n)$ iteratively by

$$
y^{(k+1)}(n)=y^{(k)}(n)-G(n) .
$$

Repeating the presented iterative procedure, the missing values are going to converge to the true signal values that produce minimal concentration measure in the DFT (sparse) domain.

\section{INSTRUMENT DESCRIPTION}

Instrument for statistical performance analysis is described in this section. The outlook of the instrument is presented in Fig.1. The proposed instrument is implemented in MATLAB, and it has two main functionalities. The first one is to test the gradient algorithm [8] in various situations by setting up all relevant parameters of input signal. The second one is to perform various statistical analysis for this algorithm. Each of these two functionalities will be explained next.

A first functionality of the instrument is to perform reconstruction for various input signals which can be made by instrument. Signals are generated by panel named "Generate input signal". Here we can create different input signals. First option is to chose signal length. There are three predefined lengths 128,256 , and 512. The next parameter which can be chosen is percent of available samples. Next, there is slider for sparsity adjustment. In this way signals with different sparsities can be created in an easy way. Also the amplitude range for signal components can be chosen. At the end, there is entry field to determine variance of noise which 


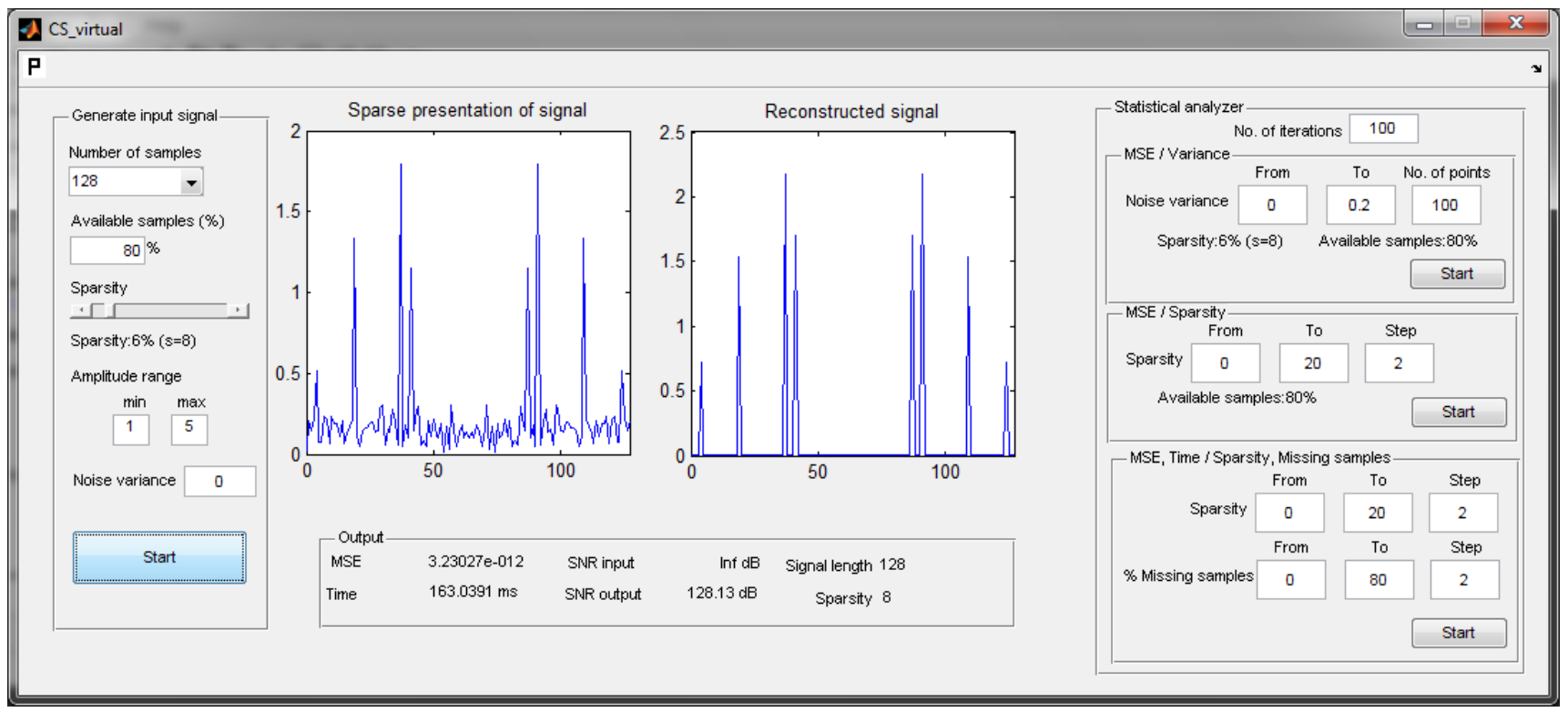

Fig. 1: Outlook of the presented instrument

will be added to available samples. When each of these parameters is changed, a new signal with appropriate setup is created. It is important to note that each signal is generated with random signal components amplitudes from specified range where number of signal components in DFT domain is specified by signal sparsity slider. After the reconstructions is performed by clicking "Start" button at the bottom of this panel, the reconstruction results are presented graphically and numerically. The DFT domains of signal with missing samples and reconstructed one are presented at the central part of instrument. Below these graphical results, there are numerical parameters describing the quality of reconstruction: MSE, time, SNR input, SNR output, length and sparsity of signal. In this way a different randomly generated signal can be tested by instrument. An example of signal reconstruction with sparsity $s=8$ and $20 \%$ of missing samples is shown in Fig.1.

The second part of the instrument is analyzer implemented in panel "Statistical analyzer". There are three separate sections to perform different statistical analysis. Note that all statistical analysis are calculated as many times as user has specified in "No. of iterations" entry field at the top of panel. Then, the obtained results are averaged before they are graphically presented.

The first part is for analyzing MSE as a function of input noise variance. The panel for setting up parameters is titled "MSE/Variance". There are three input fields where user can specify range for noise variance as well as number of points between selected range. Signal sparsity and percent of available samples is the same as the one given by "Generate input signal" panel. Clicking the "Start" button at this panel the analysis of MSE for all specified noise variances is calculated and shown graphically as it is shown in Fig.2.

The second part of analyzer is "MSE/Sparsity" panel. User

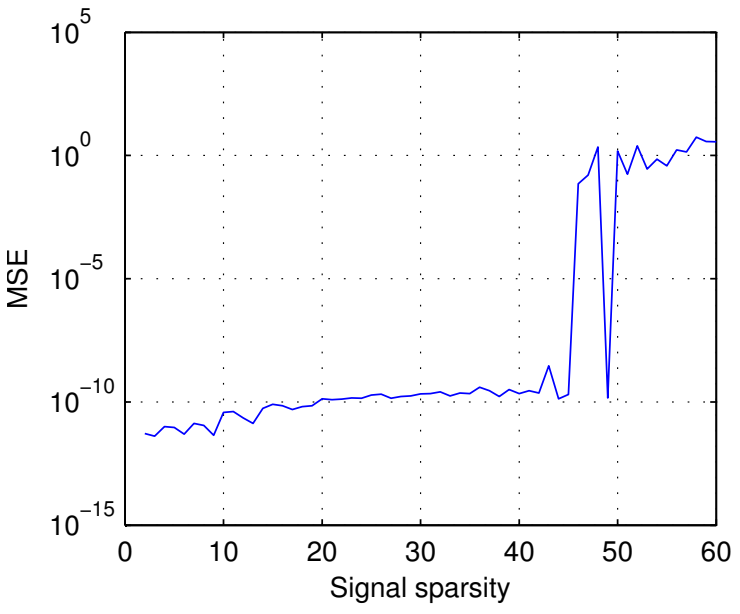

Fig. 2: MSE as a function of signal sparsity

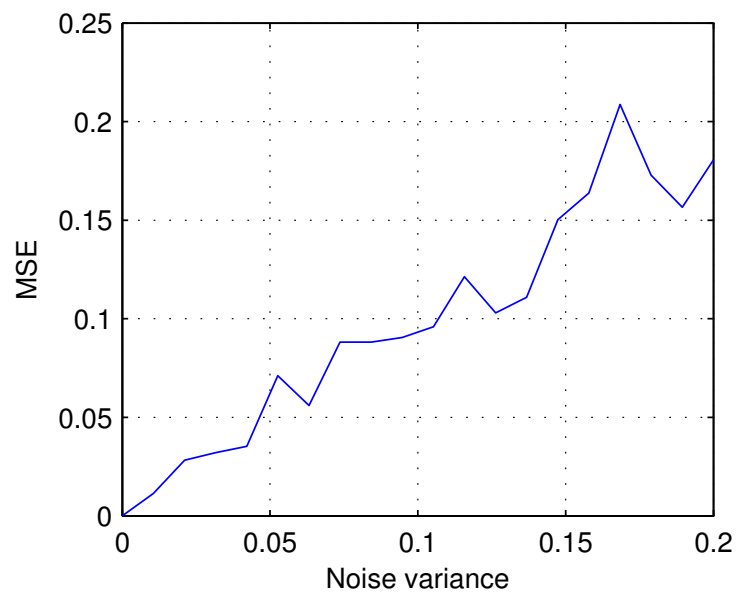

Fig. 3: MSE as a function of variance of input noise 


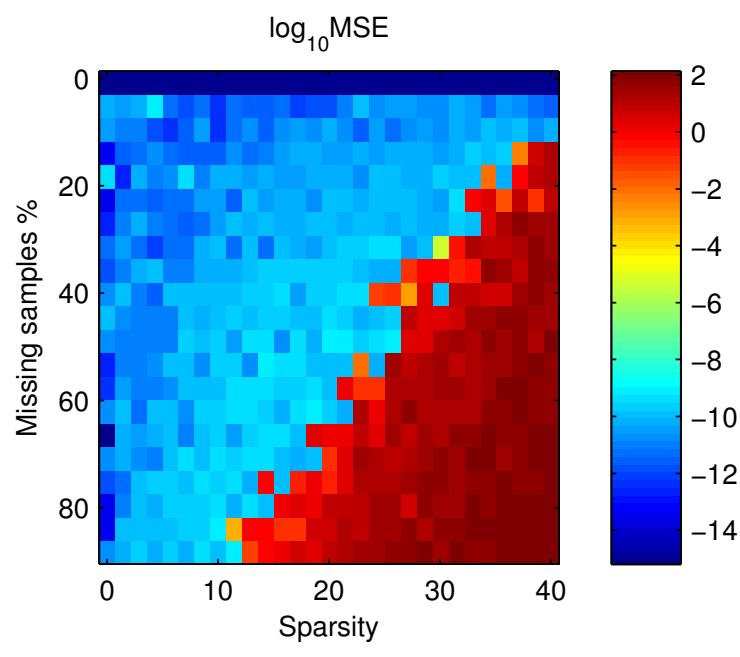

Fig. 4: MSE as a function of signal sparsity and percent of missing samples.

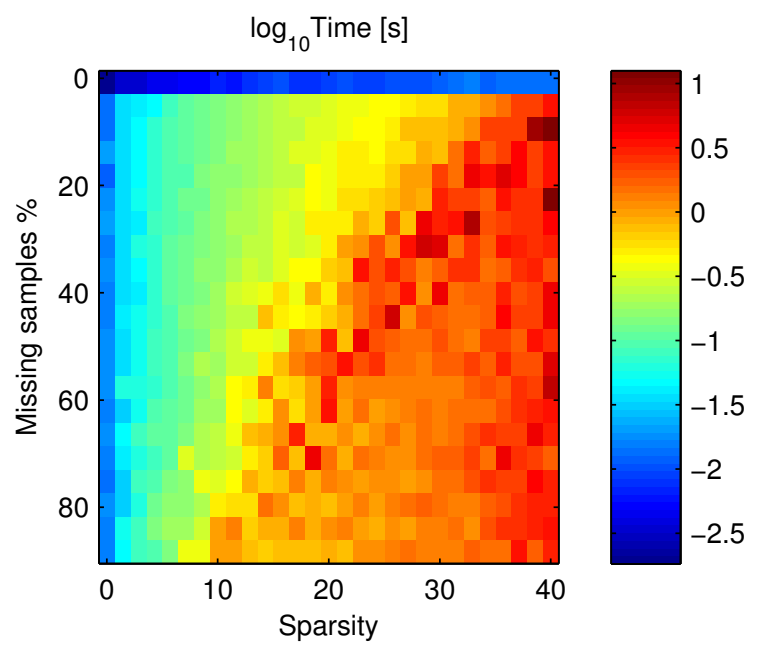

Fig. 5: Computational time as a function of signal sparsity and percent of missing samples.

can specify minimal and maximal sparsity as well as the step between considered sparsities within chosen range. The percent of available samples is the one given by "Generate input signal" panel. A new signal in each realization is generated and missing samples are reconstructed by algorithm. Average MSE for each considered sparsity is calculated (averaged) and results are shown as in Fig.3.

The third part of analyzer is for calculating average MSE and computational time for various combinations of sparsity and percent of missing samples. The results are calculated and then averaged. MSE and time as a functions of two variables (sparsity and percent of missing samples) are presented then as it is shown in Fig.4 and Fig.5.

\section{CONCLUSION}

A statistical performance analyzer for gradient based signal reconstruction algorithm is presented. To demonstrate the performance of instrument for various input signals, a special panel for setting up all relevant signal parameters is presented. In this way, signals with different lengths, sparsities and percent of available samples can be created and their reconstruction can be performed. Reconstruction results are shown graphically by presenting DFT domain of signal before and after reconstructions. Also some measurable parameters like MSE, reconstruction time, input SNR, output SNR are presented. The performance analysis based on the statistical tests are provided in the second part of the instrument. The idea of this module is to analyze the MSE in the presence of noise, then the influence of the signal sparsity and missing samples on the reconstruction quality as well as to estimate the reconstruction time for each of the tested scenarios.

\section{ACKNOWLEDGMENT}

This research is supported by the project "New ICT Compressive Sensing Based Trends Applied to Multimedia, Biomedicine and Communications (CS-ICT)" (Montenegro Ministry of Science, Grant No. 01-1002)

\section{REFERENCES}

[1] S. Stanković, I. Orović, and M. Amin, "Compressed sensing based robust time-frequency representation for signals in heavy-tailed noise," in Information Science, Signal Processing and their Applications (ISSPA), 2012 11th International Conference on, pp. 605-610, IEEE, 2012.

[2] LJ. Stanković, S. Stanković, I. Orović, and M. Amin, "Robust TimeFrequency Analysis based on the L-estimation and Compressive Sensing," 2013.

[3] D. L. Donoho, "De-noising by soft-thresholding," Information Theory, IEEE Transactions on, vol. 41, no. 3, pp. 613-627, 1995.

[4] E. J. Candès, J. Romberg, and T. Tao, "Robust uncertainty principles: Exact signal reconstruction from highly incomplete frequency information," Information Theory, IEEE Transactions on, vol. 52, no. 2, pp. 489-509, 2006.

[5] R. G. Baraniuk, "Compressive sensing [lecture notes]," Signal Processing Magazine, IEEE, vol. 24, no. 4, pp. 118-121, 2007.

[6] M. A. Figueiredo, R. D. Nowak, and S. J. Wright, "Gradient projection for sparse reconstruction: Application to compressed sensing and other inverse problems," Selected Topics in Signal Processing, IEEE Journal of, vol. 1, no. 4, pp. 586-597, 2007.

[7] G. Davis, S. Mallat, and M. Avellaneda, "Adaptive greedy approximations," Constructive approximation, vol. 13, no. 1, pp. 57-98, 1997.

[8] LJ. Stanković, M. Daković, and S. Vujović, "Adaptive variable step algorithm for missing samples recovery in sparse signals," Signal Processing, IET, vol. 8, no. 3, pp. 246-256, 2014.

[9] LJ. Stanković, "A measure of some time-frequency distributions concentration,” Signal Processing, vol. 81, no. 3, pp. 621-631, 2001.

[10] E. J. Candes and T. Tao, "Decoding by linear programming," Information Theory, IEEE Transactions on, vol. 51, no. 12, pp. 4203-4215, 2005. 